\title{
Carnets
}

Revue électronique d'études françaises de l'APEF

Première Série - 1 Numéro Spécial | 2009

Cultures littéraires : nouvelles performances \& développement

\section{L'acte de traduction : écrire, publier, lire. La traduction littéraire à l'âge de la mondialisation}

\section{Mathieu Dosse}

\section{(2) OpenEdition}

Journals

Édition électronique

URL : http://journals.openedition.org/carnets/3883

DOI : $10.4000 /$ carnets.3883

ISSN : 1646-7698

Éditeur

APEF

Édition imprimée

Date de publication : 1 juin 2009

Pagination : 243-255

Référence électronique

Mathieu Dosse, « L'acte de traduction : écrire, publier, lire. La traduction littéraire à l'âge de la

mondialisation », Carnets [En ligne], Première Série - 1 Numéro Spécial | 2009, mis en ligne le 16 juin

2018, consulté le 01 mai 2019. URL : http://journals.openedition.org/carnets/3883 ; DOI : 10.4000/ carnets.3883

Carnets est mis à disposition selon les termes de la licence Creative Commons - Atribution - Pas d'utilisation commerciale 4.0 International. 


\title{
L'ACTE DE TRADUCTION : ECRIRE, PUBLIER, LIRE \\ La traduction littéraire à l'âge de la mondialisation
}

MATHIEU DOSSE

Université Paris VIII

\begin{abstract}
Résumé
Omniprésente aujourd'hui dans la production littéraire, la traduction joue un rôle clé à tous les niveaux. Cette communication s'intéresse au changement opéré par la traduction dans trois pôles distincts de la vie de l'œuvre: sa création, sa production et sa lecture. A l'âge de la mondialisation, nous ne lisons plus et nous n'écrivons plus comme avant. Les écrivains, qui sont aussi des lecteurs, ont très tôt, pour une part d'entre eux, abandonné la visée d'une littérature nationale au profit d'une inscription dans un monde aux frontières plus lointaines. Le marché du livre a, de son côté, subi autant qu'il a contribué à ce changement, qui ne doit pas être perçu comme négatif. Car lire un texte traduit ce n'est, contrairement à l'idée reçue, lire un pis-aller. La lecture en traduction est une lecture d'autant plus active qu'elle fait appel à une interactivité plus grande de la part du lecteur.
\end{abstract}

\section{Abstract}

Translation is nowadays everywhere and it plays a great part in every step in the chain of production of the book: writing, publishing and reading. We do not read nor do we write like we did before. Writers (nevertheless readers) have turned away, as least part of them, from a national literature. Publishers are at the same time victims and actors of this new apprehension of the global literature. This is not a bad thing. Indeed, to read a translated text is not, as commonly thought, an inferior act. One might even say that to read a translation implies a greater awareness from the reader, as the translated text demands more interaction between text and reader.

Mots-clés: Traduction, Lecture, Marché du livre, Esthétique, Mondialisation

Keywords: Translation, Reader-response, Publishing industry, Aesthetics, Globalization 
Nous ne lisons pas aujourd'hui comme nous lisions il y a à peine un siècle. Un changement, qui n'est pas uniquement dû à la mondialisation, s'est peu à peu opéré ; lentement, mais sûrement, notre manière d'appréhender la littérature, notre manière de lire, au sens fort du mot, le texte littéraire, s'est modifiée. Que connaissait-on, dans la France du XIXe siècle, de la littérature étrangère ? On lisait quelques auteurs anglais, Dostoïevski avait ouvert la voie à la découverte des écrivains russes, la poésie espagnole n'était pas méconnue. Mais la publication de romans français dépassait largement en nombre celle de romans étrangers. Le plus souvent, d'ailleurs, les traductions gommaient tout caractère "étranger" du texte à traduire : il ne fallait pas que le texte traduit sente la traduction. Mais surtout, la littérature étrangère était mise à part, reléguée à la périphérie de la littérature nationale. D'un côté la littérature nationale, écrite en langue originale, de l'autre la littérature étrangère, traduite, que l'on voulait bien considérer, mais se gardant bien de l'inscrire dans le canon national. Les auteurs (qui sont également des lecteurs à part entière), s'ils lisaient des textes traduits, dialoguaient peu avec leurs contemporains étrangers.

Un changement s'est peu à peu opéré au cours du XXe siècle, pour s'accentuer à partir des années 1970. Julien Gracq, par exemple, fait part de ses lectures de Dostoïevski, mais ses auteurs, ceux avec lesquels il établit un véritable dialogue sont Chateaubriand, Stendhal et Flaubert. Maurice Blanchot lit Kafka, mais c'est encore un acte isolé ; et il le lit en allemand. Quelle est la part accordée à la littérature étrangère chez Genette ou Barthes au début des années 60 ? Les grandes études d'auteur dans ces années-là sont faites d'après des textes lus en langue originale, non des traductions.

La part de la littérature étrangère grandissant, la place accordée à la lecture de textes traduits devient plus importante. Aujourd'hui cette place, sans cesse croissante a sans doute, pour certains lecteurs du moins, atteint un niveau critique : les lectures de textes étrangers sont, en nombre, égales ou supérieures à celles de textes nationaux. On peut lire, dans un article récent que "cette année, sur 727 romans annoncés, 234 sont des traductions, soit une progression de $10 \%$ par rapport à 2006. La langue anglaise confirme sa suprématie avec 123 titres, devant l'espagnol (19), l'italien (18), les langues scandinaves (11) et l'allemand (10)" (Beuve-Méry, $2007: 11)$. Les données brutes ne font que confirmer un mouvement. II faut les prendre avec circonspection dans la mesure où elles ne concernent qu'une partie de lecteurs potentiels ; on ne lit pas que les nouveautés. II faut en outre hiérarchiser (sans aucun jugement de valeur) les lectures. Certaines lectures, dans la mesure où elles exercent une influence profonde sur les textes à venir, sont plus "importantes" que d'autres. La vie de la littérature, sa vie concrète (son lieu de production) se manifeste dans ses textes nationaux. Les lecteurs-écrivains (autrement dit, tous les écrivains, car on n'écrit pas sans avoir lu) sont des lecteurs privilégiés dans la mesure où leurs lectures se manifestent de manière concrète dans leurs œuvres. 
Gilles Barbedette décrit précisément ce tournant, opéré il y a près d'un siècle déjà :

Beckett écrivant en français et se traduisant en anglais, Borges puisant dans toutes les langues et les littératures la source de son inspiration, Nabokov écrivant d'abord en russe la moitié de son œuvre puis l'autre moitié en anglais : tout cela indique bien plus qu'une tendance au cosmopolitisme de la littérature, qui, au fond, a toujours existé. C'est le signe que nous sommes entrés dans l'Ere de l'Après-Babel et que le découpage des arts en petites cloisons nationales n'a plus aucun sens. L'idée qu'une tradition romanesque puisse se suffire à elle-même est devenue intensément absurde à l'âge de la géographie planétaire. (Barbedette, 1986 :15)

Barbedette met en avant, à demi-mot, un processus de lecture-écriture où les deux moyens de production (car lire, c'est aussi produire) sont intrinsèquement liés. Si Borges s'inspire de la bibliothèque universelle, c'est qu'il est un lecteur de cette bibliothèque ; Cervantès n'est pour lui qu'un auteur parmi d'autres. S'il s'inscrit dans une littérature résolument occidentale (malgré quelques "orientalismes"), il dépasse le cadre de la littérature hispanophone (ce n'est pas exemple pas le cas de Roberto Arlt, autre Argentin, plus ancré dans une littérature nationale, bien qu'il fût un grand lecteur de Dostoïevski). Nabokov, qui ne partage pas avec Borges le goût pour les œuvres classiques, est un grand lecteur des littératures russe, anglaise et française et il joue de façon remarquable avec les œuvres de ces trois pays.

Ecriture et lecture sont donc étroitement liées. Nous pouvons toutefois, avec Wolfgang Iser, séparer ce lieu de production en deux pôles distincts (Iser, 1976) : le pôle artistique (la création), le pôle esthétique (la réception). C'est à ces deux pôles que nous nous intéresserons, mais en y ajoutant un troisième, duquel ils dépendent, et que l'on oublie souvent : la dimension sociologique. Car le marché de la traduction, s'il fait bien entendu partie intégrante du marché de l'édition, s’il répond aux mêmes impératifs économiques, respecte des règles propres, que nous détaillerons ici.

\section{Le marché de la traduction}

Sans que l'on puisse les séparer complètement, il y a une distinction à faire entre une mondialisation de plus en plus accentuée, qui facilite les échanges et force les traductions (processus indépendant de la littérature et que cette dernière subit) et un mouvement interne aux œuvres, impulsion active prenant en compte un espace littéraire en expansion. La traduction y joue-t-elle le même rôle ? Utilitaire dans le premier cas et inscrite dans le texte dans le second, en tant processus créatif, la traduction joue en tout cas un rôle 
de premier plan. Le texte traduit fait aujourd'hui définitivement partie de la vie active des œuvres. La traduction est inscrite, ancrée, dans la création même des textes. Autrement dit, même un lecteur hypothétique n'ayant jamais lu de traduction (ne lisant que des textes nationaux et des textes étrangers en langue originale) aura quand même « lu » des traductions, tant celles-ci font partie des œuvres - et de leur survie.

"Les flux de traduction constituent un indicateur de l'évolution des échanges entre cultures" (Sapiro, 2008 : 20) nous rappelle Gisèle Sapiro dans un ouvrage consacré au marché de la traduction, avant de préciser que ces échanges ne répondent pas uniquement à une loi de marché, à un "impérialisme économique" immaîtrisable. Certes, on ne peut nier qu'aujourd'hui l'anglais domine largement la production littéraire (en traduction) mondiale. En atteste l'asymétrie des flux de traduction : on traduit bien plus de l'anglais que de toute autre langue ; et l'on traduit relativement peu en anglais. Les tableaux fournis par Sapiro (d'après des bases de données constituées principalement à partir de l'Index Translationum et du Syndicat national de l'édition - SNE) ont de quoi terrifier : il semble évident, à les regarder, que l'anglais est, chaque jour davantage, plus présent dans le marché du livre. Si ce constat vaut surtout pour des textes non littéraires, la littérature n'y échappe pas pour autant. Une grande tradition littéraire respectée ne suffit pas à expliquer cet intérêt pour la littérature anglo-saxonne. Certes un livre traduit de l'anglais vendra sans doute plus qu'un livre traduit d'une langue ou d'un pays moins connus, ou dont la littérature n'est pas reconnue. C'est là une logique mercantile que connaissent bien les éditeurs. Mais pas seulement : des enjeux politiques et idéologiques y contribuent fortement. Ces enjeux, d'ailleurs, jouent également en faveur d'une résistance à l'hégémonie. Ainsi, certains pays favorisent concrètement la traduction d'ouvrages nationaux. C'est par exemple le cas du Portugal, qui favorise à partir des années 1980 l'essor des écrivains portugais en France en participant financièrement à l'édition des traductions de António Lobo Antunes, Fernando Pessoa ou encore José Saramago (Sapiro, 2008).

Car le monde de l'édition, en France du moins, est régi par une économie qui, si elle profite sans doute aux écrivains nationaux, n'est pas toujours favorable à la littérature étrangère. Les grandes maisons d'éditions françaises publient à perte un certain nombre d'auteurs (qui, sur le long terme, contribuent à la renommée et la légitimité de la maison en question). Cette perte est compensée par la vente de best-sellers, rentable à court terme. Ce système permet la publication d'auteurs qui autrement n'auraient aucune chance d'être publiés. Mais qu'en est-il des traductions ? Certaines maisons d'édition comme Métailié ou Actes Sud se sont spécialisées dans la traduction ; d'autres, comme les éditions de Minuit, n'en publient jamais. Tant pour les maisons spécialisées que pour les éditeurs publiant des traductions et des originaux, le constat reste le même : il est financièrement risqué de publier une traduction. Celle-ci, en effet, coûte plus cher à produire qu'un ouvrage national, et même 
si certaines traductions savent rencontrer leur public, il est risqué de publier des auteurs dont le pays d'origine est méconnu. Sans une aide financière du pays en question (que ce soit pour des raisons économiques ou idéologiques, certains pays ne favorisent pas la traduction de leurs auteurs), peu d'écrivains auront la chance de connaître une vie en traduction. Constat que partage ce directeur de collection :

Peut-être que si [Yaakov] Shabtaï avait été allemand ou américain, il aurait eu beaucoup plus de succès, mais étant donné justement qu'il a écrit dans une langue si peu connue et reconnue, il n'a peut-être aucune chance, en fait. Comme Agnon, d'ailleurs, il n'a aucune chance, Agnon est pour moi un des plus grands écrivains du $20^{\mathrm{e}}$ siècle, vraiment à l'égal de Thomas Mann, Musil, enfin vraiment, Broch, etc. des très très très très grands, et il est à peine connu, et quand il est connu, il est mal traduit, enfin, bon. Et ça je me demande si ce n'est pas dû en fait à la langue, tout simplement, dans laquelle il écrit, quoi, à l'hébreu, au statut de ces langues-là, au statut fragile, minoritaire de ces langues-là (entretient du 6 juin 2002 ; directeur de collection d'une maison indépendante de taille moyenne) (Sapiro, $2008: 200$ )

Constat terrible, mais que nous devons prendre en compte lorsque nous analysons la vie et la survie des œuvres. Toute littérature nationale est bien sûr également soumise aux lois du marché de l'édition, certains auteurs de génie ne trouveront peut-être jamais d'éditeur, ou ne seront pas suffisamment soutenus pour être découverts par leurs hypothétiques lecteurs. Mais les textes traduits peinent davantage car, comme nous l'avons $\mathrm{vu}$, ils font les frais de la politique culturelle ou de la relative impuissance de leur pays de provenance. Nous devons nous en souvenir lorsque nous analysons la portée d'une œuvre donnée à un moment donné. Nous savons bien qu'il existe de chef d'œuvres méconnus du grand public parce qu'ils n'ont pas trouvé d'éditeur, mais le constat est d'autant plus attristant lorsque nous comprenons que c'est toute la « chaîne de production » qui est pour ainsi dire altérée.

\section{Le pôle artistique : écrire à l'ère de la traduction}

"L'ère de la traduction" est une expression qui a de quoi inquiéter. Entrerait-on dans un âge où, à défaut de parler la même langue, nous serions en train privilégier la communication avant toute autre forme de d'expression ? Communiquer, au sens péjoratif du mot, c'est transmettre un contenu détaché de toute forme. En réalité, l'ère de la traduction a toujours existé. Nous avons toujours traduit ; l'occident du moins s'est construit par la traduction. L'histoire de la traduction remonte à la Bible traduite de l'hébreu en grec. La plupart les moments clés de notre civilisation ont été des grands moments de traduction : les 
Latins traduisaient les Grecs ; la Renaissance a également connu un nombre important de traductions. Si l'Orient n'a pas connu la même histoire, il ne faut pas oublier que les Chinois, vers le début du XXe siècle ont commencé à traduire abondamment les romans français et anglais, poussés sans doute par une soif de connaissance et une curiosité bienveillante, mais également dans le but très politique de s'enrichir, au sens noble du mot. Or, aujourd'hui, à l'heure de la mondialisation, c'est l'écriture même, la production artistique qui a changé.

N'y voyons pas uniquement un but commercial. Certes, certains écrivains, dans le but de gagner un marché plus grand, écrivent en pensant déjà à la réception de leur texte à l'étranger. Mais d'autres inscrivent dans leur poétique propre, la traduction. II ne s'agit pas tant de vendre plus: pour les lecteurs de textes traduits, pour des grands lecteurs de traduction, écrire sans tenir compte de la traduction semble difficilement concevable.

Irions-nous jusqu'à dire que la langue de tels textes est moins travaillée ? Ce serait croire que l'on puisse séparer sens et forme, comme si la langue ne faisait pas partie intégrante d'une poétique. Nabokov par exemple écrit dans un anglais extrêmement précis et difficilement traduisible, mais il inscrit son texte dans un univers décontextualisé, comme celui d'Ada ou l'ardeur, où trois langues (l'anglais, le russe et le français) sont imbriquées dans une «Anti-Terra " qui rappelle seulement l'Amérique du Nord, mais dont les toponymes ne font référence qu'à la littérature. Même Lolita, son roman le plus contextualisé (car c'est aussi un roman sur l'Amérique des années 50) n'échappe pas à la l'intertextualité foisonnante, qui comprend les chefs-d'œuvre de la littérature occidentale.

Dès ses premiers écrits, en russe, Nabokov, contrairement à d'autres écrivains de l'immigration russe, s'inscrivait déjà dans une littérature universelle, et non dans une littérature dite "nationale". Cette littérature universelle était bien entendu une littérature occidentale, et non mondiale, et Nabokov ne fait que suivre l'exemple d'autres très grands auteurs avant lui. II y a peut-être pourtant une spécificité actuelle, une propension grandissante d'auteurs à tenir compte de la traductibilité de leur texte. Pour Sapiro, il s'agit de "stratégies d'universalisation" qui peuvent prendre les formes les plus diverses et qui mettent en cause l'autonomie des littératures nationales.

Les formes d'universalisation varient des plus politisées aux plus dépolitisées. Le mode d'universalisation politisé souligne la dimension morale ou encore la dimension historique et mémorielle de la littérature. C'est le cas, historiquement, au Seuil et depuis les années 1980, chez Fayard, où l'on valorise par exemple, dans l'œuvre d'un auteur comme Camilleri, l'affirmation d'une "singularité culturelle", d'une tradition culturelle spécifique, mais en tant qu'expérience inscrite "dans un universel", "dans un universel mondialisé", et non comme particularisme enfermé "dans des dialectes". Cet 
auteur est également publié par Anne-Marie Métailié, laquelle met en avant de manière générale l'humanisme qui fait la "vocation et l'universalité" d'œuvres pourtant ancrées dans une réalité sociale et politique très concrète, qu'il s'agisse de décolonisation, du passé dictatorial en Amérique latine, ou de l'expérience du communisme en Allemagne (Sapiro, 2008 : 207)

On le voit, le problème se complexifie lorsque nous prenons en compte des écrivains à vocation hautement universelle, mais dont la poétique repose sur des référents très ancrés dans un lieu ou un temps donné. João Guimarães Rosa, par exemple, dont la traduction est extrêmement ardue, tant le texte original repose sur la connaissance d'un territoire particulier (et ce, même si la langue de l'auteur n'est en rien une copie du portugais parlé dans le sertão brésilien, mais doit être davantage perçue comme une langue littéraire qui utilise des possibilités virtuelles d'une langue existante). Au-delà de la simple traductibilité de la "langue" de Guimarães Rosa (dont le vocabulaire et la syntaxe différent du portugais, même de celui parlé au Brésil), c'est tout un "univers", peu connu en Europe, qu'il faudrait traduire d'une façon ou d'une autre, sans l'exotiser et sans pour autant le transposer par une hypothétique équivalence. Guimarães Rosa, s'il y incluait une part importante d'universalisation, ne tenait visiblement pas compte de la "traductibilité" de ses textes. José Saramago, au contraire, même s'il parle dans tous ses livres du Portugal, prend soin, par exemple dans As intermitências da morte, de brouiller des référents trop explicites. Ses textes, même lorsqu'ils sont satyriques, s'inscrivent d'emblée dans une littérature universelle. Comment ne pas penser également à Antoine Volodine définissant son œuvre comme une "littérature étrangère écrite en français" ? Ou encore Agota Kristof, écrivain hongrois d'expression française, qui écrit dans une langue extrêmement dépouillée, facilement traduisible (ce qui n'enlève en rien ses qualités littéraires) ?

Il semble donc bien que la mondialisation ait laissé des traces profondes chez certains écrivains, mais il ne faudrait pas pour autant en surestimer la portée. Certains grands romans contemporains sont écrits dans une langue et avec des référents très contextualisés. On ne peut nier en revanche que même ces textes s'inscrivant dans une littérature nationale sont le fruit d'une lecture décentrée. Car un écrivain ou un lecteur qui ne lirait que des textes issus de la littérature nationale, ne serait-il pas aujourd'hui un anachronisme vivant, pour reprendre l'expression d'Alain Broucys ?

\section{Le pôle esthétique : lire en traduction}

Les modalités de lecture varient en fonction du lieu et du temps dans lequel elles se situent. Ainsi, comme le signale Lawrence Venuti, Anglais et Américains lisent peu de 
traductions: $2 \%$ seulement des publications annuelles sont des traductions en 1990 . Venuti cite, comme contre-exemple, la France qui compte $12 \%$ de traductions, et l'Italie, avec 25,4\%. On traduit surtout de l'anglais. En 1983, l'anglais est largement en tête parmi les langues traduites dans le monde (24.468 ouvrages traduits de l'anglais). Le français et le russe arrivent en deuxième avec près de 6000 ouvrages, suivis de près par l'allemand avec près de 5000 (Venuti, $1995: 12$ ). Ces données vieilles de plus de 20 ans montrent une hiérarchie, encore vraie aujourd'hui. II faut en tenir compte lorsque nous lisons les théoriciens anglophones tels que Venuti ou Emily Apter. Car si la place du français est particulière - dans la mesure où il est passé du statut de langue traduite à celui de langue traduisante - l'anglais souffre aujourd'hui d'un statut de langue dominante peu enviable, car foncièrement ethnocentriste (ce qui, en ce qui concerne la traduction, n'est jamais positif).

Face à ces nouvelles modalités de la lecture, tout théoricien de la lecture devrait s'interroger sur la lecture du texte traduit. Ce n'est pourtant pas le cas. Du moins, le texte traduit n'est compris dans sa spécificité. Wolfgang Iser, par exemple, s'il aborde parfois la lecture de traduction, ne fait pas de distinction entre cette dernière et un original. La traduction est lue comme un original. Les théoriciens de la lecture n'ont en effet que très rarement convoqué la traduction (sous sa forme particulière, celle de texte traduit, lu comme traduction, non comme nouvel original ou texte équivalent, comme détaché de tout original, indépendant. Lorsqu'on lit une traduction sans dire que ce qu'on lit en est une, on perd en partie la spécificité de la traduction). La raison d'une telle mégarde est à chercher du côté d'une crainte - fondée - sur la pluralité qu'implique la prise en compte de la lecture du texte traduit. Pour les tenants d'un sens unique, comme I.A. Richards ${ }^{1}$ par exemple, la prise en compte d'une lecture de traduction fausserait définitivement toute possibilité d'unification d'une seule vraie lecture (Richards, 1929). Richards est déjà bien en peine d'accepter une certaine variation entre les lectures d'un même texte ; il ne pourrait que blâmer la traduction, dont la lecture ne pourra pas être identique à celle de l'original.

Or, nous le disions plus haut, notre manière de lire a changé. Nous lisons beaucoup, peut-être de plus en plus, de traductions. Qu'est-ce que cela implique ? Faisons-nous attention aux mêmes choses lorsque nous lisons un original ou une traduction ? Sans doute pas. Du moins lorsque la traduction ne sent précisément la traduction, lorsqu'elle ne cache pas son identité propre de traduction. Une traduction qui se ferait passer pour un nouvel original tendrait à annuler les bienfaits du texte traduit. Car il y a bien un gain à lire en traduction. II est communément admis qu'un original est toujours supérieur à sa traduction. Celle-ci n'en serait, au mieux, qu'une bonne copie. Or, la traduction n'est pas seulement

\footnotetext{
${ }^{1}$ L'un des premiers théoriciens de la lecture, procédant de manière plus intuitive que méthodique, I.A. Richards réalise en 1929 une série de tests de lecture, au bout desquels il conclut que les lectures sont majoritairement fautives, mais qu'on peut remédier à cette tare par un travail de lecture assidu. II n'y a donc pour Richards qu'un seul sens possible à un texte donné.
} 
"passage" entre les cultures, ses bienfaits, si l'on peut dire, se situent à un niveau plus profond, aux portes même de la lecture. C'est pourquoi, pour comprendre ce qu'est une traduction, ce que peut faire une traduction, il faut avant tout s'interroger sur ce qu'est la lecture du texte traduit.

Traduction et lecture. Deux pratiques, deux approches théoriques qui semblent d'évidence se compléter, s'éclairer mutuellement. Rares sont pourtant les travaux qui abordent les problèmes théoriques de la traduction littéraire sous l'éclairage des théories de la lecture. Deux raisons à cela, peut-être : il y a d'abord, sans doute, une certaine méfiance de la part des théoriciens de la traduction vis-à-vis des théories de la lecture, comme si cette approche du texte ne pouvait que conduire implicitement la théorie de la traduction vers une « traductologie méthodologique » dont la finalité serait la constitution de préceptes traductifs objectifs, fondés sur les réactions empiriques des lecteurs. Cette méfiance est peut-être accrue par une méconnaissance mutuelle : c'est en Angleterre et aux Etats-Unis que les théories de la lecture ont pris leur véritable essor (après une naissance en Allemagne, à Constance) ; c'est en France que les théories de la traduction ont trouvé leur expression la plus convaincante, notamment chez Antoine Berman et Henri Meschonnic (mais aussi, d'une manière plus générale, chez nombreux traducteurs, dont le travail de traduction est souvent accompagné d'une réflexion sur celui-ci. Cette effervescence - qui n'a pas son pareil dans d'autres pays européens - tient sans doute à l'histoire de la langue française, qui a dû faire face à un renversement sans précédent au cours du vingtième siècle, passant de langue majeure, qui est traduite, à langue mineure, qui traduit). Mais peut-être y a-t-il aussi, de la part des théoriciens de la traduction, une volonté moins avouée de mettre le lecteur hors-jeu: la prise en compte de cette instance complexifie toute position théorique sur la traduction.

Car il ne suffit pas de dire, avec Meschonnic (qui reste le plus grand penseur actuel de la traduction) qu'il faut "traduire ce que font les mots" (Meschonnic, 1999). Les mots font, certes, mais le lecteur lit. Autrement dit, la constitution de l'œuvre dépend de l'interaction entre le texte et le lecteur : le lieu de l'œuvre littéraire est celui où se rencontrent texte et lecteur. Le rôle du lecteur ne peut se borner à celui d'un simple "récepteur" ou "destinataire", termes assez répandus en traductologie. Le lecteur s'implique aussi en lisant, il s'inscrit dans le texte en tant que sujet. Ce qui ne veut pas dire qu'il y ait "autant de textes que de lecteurs", mais le texte offre des schémas solides que le lecteur va concrétiser, notamment en remplissant par ses propres représentations les failles, les blancs du schéma textuel.

On voit déjà à quel point le concept traditionnel opposant des traducteurs "sourciers"à des traducteurs "ciblistes" (concept proposé par Jean-René Ladmiral et repris constamment dès que l'on parle de théorie de la traduction) perd de sa pertinence. Cette répartition dichotomique voudrait qu'il y ait d'un côté des "sourciers", qui cherchent à préserver le signifiant de la langue-source, de l'autre des "ciblistes", ne tenant compte que du sens à 
transmettre dans la langue-cible. Or, une telle répartition reste prisonnière d'une conception de la littérature où le sens serait une donnée stable, que l'on pourrait détacher de la lettre du texte, afin de la faire passer, intacte, dans une autre langue. Elle ne tient pas compte du lecteur, de sa participation dans la constitution de l'œuvre, et donc du sens. Le conflit des interprétations est pour les théories de la lecture une approche dépassée. Quant aux prétendus "sourciers", se pourrait-il que, comme le veut Ladmiral, ils n'aient de regard que pour le texte original, "qui tend à être investi comme un texte sacré" ? (Ladmiral, 1979) Non, bien sûr. Là encore, on oublie que le texte traduit est lu, que la relation entre original et traduction, aussi intime soit-elle, comme le dit Benjamin, ne peut pas être une relation de servitude, qui verrait dans le texte traduit, un texte inférieur par essence à l'original.

Penser la lecture du texte traduit, c'est aussi penser le rapport entre original et traduction. Ce rapport est-il seulement un rapport de filiation? Certes, on ne peut nier qu'une traduction ne doive son existence qu'à un original duquel, littéralement, elle naît. La traduction, parce qu'elle est mortelle, permet à l'original une survie qu'il n'aurait pu espérer sans elle (les langues, en effet, finissent par mourir, tout comme meurent les traductions et la langue dans laquelle est écrit l'original). Mais est-ce sa seule finalité ? Ne peut-on pas concevoir que la traduction, parce qu'elle est texte, non seulement nous dit quelque chose d'essentiel sur l'original (c'est une évidence) mais encore transforme cet original ? Le trajet ne se fait pas uniquement de l'original à la traduction ; les passages sont circulaires. Car la lecture agit sur le texte de même que le texte agit sur le lecteur.

Or, l'original est lu par le traducteur. Cette affirmation est si évidente, si anodine en apparence, qu'elle a de tout temps été oubliée par les penseurs de la traduction (sauf lorsqu'il s'agit de dire, sur le ton du reproche, comme le fait Paul de Man à propos de Gandillac traducteur de Benjamin , que tel traducteur a "imprégné" telle traduction de sa lecture de l'original). Placer le lecteur au cœur des enjeux de la traduction implique que l'on tienne compte du sujet traducteur. La théorie de l'effet esthétique vient bousculer ici les théories traditionnelles de la traduction qui voudraient que le lecteur soit neutre, effacé. En tant que lecteur, le traducteur inscrit sa lecture dans la traduction de l'original.

La traduction vient à son tour complexifier la théorie de l'effet en y introduisant cette troisième instance, le traducteur, dont elle ne sait pas vraiment quoi en faire. L'un de fondements de la théorie de l'effet repose sur la relation auteur/lecteur, le texte étant l'arène où ces deux instances s'affrontent en un combat singulier. Introduire une troisième instance, le traducteur, dans ce rapport dialectique, nous permet d'explorer de nouveaux domaines jusqu'alors ignorés par les théories de la lecture en général. L'affinement théorique est double : l'acte de lecture éclaire les théories de la traduction ; l'acte de traduction précise à son tour les théories de la lecture. 
Qu'en est-il du lecteur du texte traduit ? Lecteur second par définition, on cherche à le réduire au silence ; comme si sa lecture était une aberration, comme s'il n'avait pas vraiment lu, comme si, enfin, le texte traduit ne pouvait être qu'une introduction, un passage vers un original qui resterait la finalité de toute lecture. Le concept de traduction en tant que passage accentue la négativité de la traduction, ce qui fait d'elle une perte, un pis-aller. Ne peut-on pas concevoir que la traduction n'est pas que perte ? Peut-on aussi penser le texte traduit comme gain ? C'est notre proposition. Or, postuler que la traduction est un gain implique désacraliser, du moins momentanément, le texte original. Mais cette position ne doit pas être poussée dans ses extrémités. Penser le texte traduit n'implique pas vouloir brûler les originaux, comme le faisaient les latins. Brûler l'original - c'est à dire, concrètement, aujourd'hui, ne pas tenir compte du fait que ce qu'on lit est bien une traduction - c'est effacer la différence entre traduction et original, c'est détruire ce qui fait précisément la spécificité de la traduction. Cette spécificité est celle d'être une activité plurielle et temporelle.

La traduction est en effet une expérience de la pluralité. Pensons à la position décentrée du lecteur du texte traduit : traduction et original s'inscrivent dans deux mondes culturaux et linguistiques hétérogènes, régis par des "répertoires" (intertextualité, normes sociales et politiques, contextes socioculturels...) différents. Dès lors, lecture du texte original et lecture du texte traduit ne peuvent être identiques. On ne peut par conséquent que mettre fortement en doute l'idée selon laquelle on pourrait comparer le lecteur du texte original et le lecteur du texte traduit, trouver des équivalences entre les deux. Dire que la lecture d'un texte (déjà pluriel, puisque doublé par la traduction) par deux lecteurs de cultures différentes doit être la même, comme le prétend Nida, c'est avoir, nous l'avons souligné, une conception restrictive de la lecture : à l'intérieur d'une même culture, déjà, un texte ne peut se limiter à une seule interprétation. Traduire selon des équivalences, c'est ne pas tenir compte du lecteur, c'est croire que l'on peut avoir emprise totale sur le texte. A la différence du lecteur du texte original, dont le répertoire mono-culturel n'est pas remis en cause par le texte, le lecteur du texte traduit doit faire face à un espace de représentation différent (c'est le cas du moins lorsque le texte traduit ne répond pas à une visée traductive trop ethnocentrique qui réduirait toute "étrangeté" du texte original, qui effacerait en somme la spécificité plurielle de la traduction ; comme par exemple ces traductions desquelles on dit, élogieusement, "que rien en elles ne laissent deviner qu'il s'agit de traductions"). II est communément accepté que le texte traduit ne peut être que "moins beau" que le texte original. Or, nous voyons ici que ce n'est peut-être pas tout à fait le cas. L'interaction entre le texte traduit et son lecteur sera, d'une certaine manière, plus importante que celle entre le texte original et son lecteur (en effet, les éléments du répertoire du lecteur du texte traduit seront plus sollicités, ce qui favorise l'implication du lecteur dans la constitution de l'œuvre). L'interaction entre le texte et le lecteur est sans doute, des dynamiques qui gouvernent l'acte 
de lecture, celle qui contribue le plus à l'effet esthétique. On pourrait donc dire - mais c'est bien sûr une position purement théorique - que le texte traduit non seulement n'est pas "moins beau" que le texte original, mais qu'il l'est davantage.

Conclusion extrême, qui ne tient pas compte de tous les paramètres de la lecture, mais qui a le mérite de prendre le contre-pied de la doxa, encore valable aujourd'hui, selon laquelle la traduction est un mal que nous devons subir. Nous avons essayé de montrer que c'est précisément la traduction qui restructure aujourd'hui les lieux de production de la littérature, et qu'il ne faut pas y voir un autre méfait induit par la mondialisation, mais au contraire un gain apporté par cette dernière. 


\section{Bibliographie}

APTER, Emily (2006). The translation zone: a new comparative literature, Princeton : Princeton University Press

BARBedetTe, Gilles (1986), Présentation du dossier consacré à Nabokov, Magazine Littéraire, $n^{\circ} 233$, sept. 1986

Beuve-Mery, Alain (2007), "Le Monde des livres", supplément du journal Le Monde du vendredi 14 septembre 2007

ISER, Wolfgang (1976). L'acte de lecture. Théorie de l'effet esthétique. Tr. E. Sznycer, Bruxelles : Pierre Mardaga

LADMIRAL, Jean-René (1979). Traduire : théorèmes pour la traduction. Paris : Gallimard

Meschonnic, Henri (1999). Poétique du traduire. Lagrasse : Verdier

RICHARDS, I.A. (1929). Pratical Criticism. A study of literary judgment, Londres: Routledge

SAPIRO, Gisèle (dir.) (2008). Translatio. Le marché de la traduction en France à l'heure de la mondialisation. Paris : CNRS Editions

VENUTI, Lawrence (1995). The translator's invisibility. A history of translation. Londres/New York: Routledge 